Algebra univers. 52 (2004) 527-540

0002-5240/04/040527 - 14

DOI $10.1007 / \mathrm{s} 00012-004-1901-1$

(C) Birkhäuser Verlag, Basel, 2004

Algebra Universalis

\title{
The quantale of Galois connections
}

\author{
Jorge PicAdo
}

\begin{abstract}
Galois connections were originally expressed in a contravariant form with transformations that reverse (rather than preserve) order. Nowadays its covariant form (as residuated maps) is more often used since it is more convenient; namely compositions of residuated maps are handled more easily. In this paper we show that this is not a serious disadvantage of the contravariant form (at least in the natural context for uniform structures, where we need it), by introducing an operation of composition in the complete lattice $\operatorname{Gal}(L, L)$ of all (contravariant) Galois connections in a complete lattice $L$, that allows us to work with Galois connections in the same way as one usually works with residuated maps. This operation endows $\operatorname{Gal}(L, L)$ with a structure of quantale whenever $L$ is a locale, allowing the description of uniform structures in terms of Galois connections.
\end{abstract}

\section{Introduction}

Originally Galois connections were expressed in a symmetric but contravariant form with transformations that reverse order (early references to this form are [3], [6] and [17]). A Galois connection [3] between the partially ordered sets $A$ and $B$ is a pair $\left(f, f^{+}\right)$of order-reversing (antitone) maps $f: A \rightarrow B$ and $f^{+}: B \rightarrow A$ such that

$$
a \leq f^{+} f(a) \text { for all } a \in A \text { and } b \leq f f^{+}(b) \text { for all } b \in B
$$

or, equivalently,

$$
a \leq f^{+}(b) \text { iff } b \leq f(a) \text { for every } a \in A \text { and } b \in B .
$$

$\operatorname{Gal}(A, B)$, also denoted $A \otimes B[22]$, is the set of all Galois maps between $A$ and $B$, that is, all antitone maps $f: A \rightarrow B$ for which there is $f^{+}: B \rightarrow A$ such that $\left(f, f^{+}\right)$is a Galois connection, with the pointwise partial order. Galois connections appear in many areas of mathematics (cf. the survey [5]).

Presented by J. Adámek.

Received August 18, 2004; accepted in final form September 13, 2004.

2000 Mathematics Subject Classification: 06A15, 06D22, 06F07, 54E15.

Key words and phrases: Galois connection, residuated map, complete join-semilattice, frame, locale, quantale, quantic nucleus, uniform locale, polarity, axiality.

Research supported by the Centro de Matemática da Universidade de Coimbra and by the Fundação para a Ciência e Tecnologia through grant FCT POCTI/1999/MAT/33018 "Quantales". 
Nowadays many authors prefer to work with Galois connections in the covariant form because of its convenience (the survey [5] contains a list of references to this form). In order to avoid confusion we refer to this dualized form as a residuated pair: a residuated pair between the partially ordered sets $A$ and $B$ is a pair $(f, g)$ of order-preserving maps $f: A \rightarrow B$ and $g: B \rightarrow A$ such that

$$
a \leq g f(a) \text { for all } a \in A \text { and } f g(b) \leq b \text { for all } b \in B .
$$

The map $f: A \rightarrow B$ in a residuated pair $(f, g)$ is called residuated and the map $g: B \rightarrow A$ is called residual. Thus $f$ is residuated (resp., residual) if and only if $f \in \operatorname{Gal}\left(A, B^{o p}\right)$ (resp., $\left.f \in \operatorname{Gal}\left(A^{o p}, B\right)\right)$.

As Blyth and Janowitz (in their monograph on Residuation Theory [4], p. 19) and Erné, Koslowski, Melton and Strecker (in [5]) remarked, the reason for preferring residuated maps rather than Galois connections can be found in the fact that compositions of residuated pairs are handled more easily: two residuated maps may be composed to yield a new residuated map and this is not the case with antitone maps. Indeed, if $f: A \rightarrow B$ and $g: B \rightarrow C$ are residuated maps, then $g f$ is clearly residuated with $(g f)^{+}=f^{+} g^{+}[4]$. This composition endows $\operatorname{Gal}\left(A, A^{o p}\right)$ with a structure of quantale (complete residuated semigroup). We call it the quantale of residuated maps.

But there remains the question: how to compose Galois connections directly, in order to get a structure of quantale for $\operatorname{Gal}(A, A)$ ? This is the problem which motivated us to write this paper. Its relevance comes from the fact that this quantale reveals to be an important tool in the entourage-like approaches to uniform structures (cf. [7]). In this paper we present a composition operation $\circ$ for Galois maps. Instead of presenting a direct proof that $(\operatorname{Gal}(A, A), \circ)$ is a quantale by proving the associativity of $\circ$ and the distributivity of it over joins in $\operatorname{Gal}(A, A)$, we prefer to follow an indirect but more general and elegant approach, by showing that $\operatorname{Gal}(A, A)$ is a quotient of the set $\operatorname{Ant}(A, A)$ of all antitone maps from $A$ to itself, expressed in terms of a suitable quantic nucleus. This way $\operatorname{Gal}(A, A) \operatorname{simply}$ inherits the algebraic properties from the more general and simpler $\operatorname{Ant}(A, A)$, and we avoid the technical difficulties inherent to proving associativity of $\circ$ and the distributivity of it over joins.

A more detailed description of this paper is as follows. We begin by recalling, in Section 2, some general background about frames, locales, quantales and Galois connections. We also fix terminology and notation that will be used in later sections. In the third section we introduce the composition operation $\circ$ for Galois connections and in Section 4 we introduce an auxiliary operation for antitone maps and prove that this is an associative operation when we specialize to frames. Then we show that this operation endows $\operatorname{Ant}(A, A)$ with a structure of quantale whenever $A$ is a frame (Proposition 4.4). In Section 5 we recall some facts about quantic 
nuclei and, as a useful intermediate step, we introduce the notion of quantic prenucleus extending the definition of localic prenucleus of [1]. These facts allow us, in Section 6 , to conclude from Proposition 4.4 that $(\operatorname{Gal}(A, A), \circ)$ is also a quantale. In the last section we illustrate the importance of this quantale with a brief survey describing its application to uniform structures effected in [7]. Namely we refer that the theory of uniform structures (both classical and pointfree) may be completely described in terms of Galois connections and conclude that the equivalence between the somewhat different approaches to uniform locales of [8] and [20] is a special case of a very general phenomenon on Galois connections.

\section{Background}

In this section we introduce terminology and notation along with an overview of the construction of tensor products of complete join-semilattices, presented in the way which is appropriate for its application in the later sections.

2.1. Frames and locales A frame is a complete lattice $L$ in which the infinite distributive law

$$
x \wedge \bigvee S=\bigvee\{x \wedge s \mid s \in S\}
$$

holds for all $x \in L$ and $S \subseteq L$. Frames are exactly complete Heyting algebras with the Heyting implication given by $x \rightarrow y:=\bigvee\{s \in L \mid x \wedge s \leq y\}$.

Frame homomorphisms are defined as maps between frames which preserve finitary meets (including the top element $1:=\wedge \emptyset$ ) and arbitrary joins (including the bottom element $0:=\bigvee \emptyset)$. The resulting category is called the category of frames. The study of frames was motivated by the following observation. Let $X$ be a topological space and let $\mathcal{O}(X)$ be the complete lattice of open subsets of $X$. Since finite meets and arbitrary joins correspond to the set-theoretic operations of intersection and union, $\mathcal{O}(X)$ is a frame. Moreover, if $f: X \rightarrow Y$ is a continuous map of spaces, the induced map $f^{-1}: \mathcal{O}(Y) \rightarrow \mathcal{O}(X)$ is a frame homomorphism. The functor $X \mapsto \mathcal{O}(X), f \mapsto f^{-1}$ is contravariant and therefore the category of "generalized spaces" [12] should be the opposite of the category of frames. The objects of this category are called locales and morphisms locale maps. Thus locales form an order-theoretic counterpart of topological spaces. Locales were introduced by J. Isbell [12] to provide a better categorical context for topology.

For more information on frames and locales we refer the reader to [13].

2.2. Quantales In the definition of a frame, by replacing the commutative operation $\wedge$ with a general associative binary operation, one obtains a quantale (a 
"non-commutative generalized space"). Thus a quantale is a complete lattice $Q$ equipped with an associative multiplication - which distributes over arbitrary joins:

$$
x \cdot \bigvee S=\bigvee\{x \cdot s \mid s \in S\} \text { and }(\bigvee S) \cdot x=\bigvee\{s \cdot x \mid s \in S\}
$$

for all $x \in Q$ and $S \subseteq Q$. A quantale homomorphism is a map between quantales which preserves arbitrary joins and the multiplication.

The term quantale was suggested by C. J. Mulvey [15] as a "quantization" of the term locale. Its definition provides a non-commutative extension of the concept of locale. The intention was to develop the concept of non-commutative topology, introduced by R. Giles and H. Kummer [9], while providing constructive foundations for quantum mechanics and non-commutative logic. Further details about quantales can be found in [18] and [21].

2.3. Galois connections Let $A$ and $B$ be complete lattices. In this case, as it is well-known (and very easy to prove), maps $f$ and $f^{+}$in a Galois connection uniquely determine each other; indeed

$$
f(a)=\bigvee\left\{b \in B \mid f^{+}(b) \geq a\right\} \text { and } f^{+}(b)=\bigvee\{a \in A \mid f(a) \geq b\} .
$$

Furthermore, $f \in \operatorname{Gal}(A, B)$ if and only if

$$
f(\bigvee S)=\bigwedge f(S) \text { for every } S \subseteq A \text { (in particular, } f(0)=1 \text { ), }
$$

that is, if and only if $f$ is a complete join-morphism $A \rightarrow B^{o p}$. The set $\operatorname{Gal}(A, B)$ is also a complete lattice (meets are just defined pointwise). On the other hand, $f: A \rightarrow B$ is residuated if and only if it is a join-homomorphism.

For a more categorical approach to Galois connections we refer to [11].

2.4. Tensor products Let $A$ and $B$ be complete join-semilattices. As Shmuely proved in [22], $\operatorname{Gal}(A, B)$ is the tensor product of $A$ and $B$ in the category of complete join-semilattices (whose objects are complete join-semilattices, that is, complete lattices, and morphisms are maps preserving arbitrary joins). This is the analogue for complete join-semilattices of the construction of tensor products of modules over a commutative ring (cf. [14]). There is an equivalent way of describing the tensor product of complete join-semilattices, which will be very useful in the next section. As Shmuely proved ([22], Theorem 1.3; see also [10]), the elements of $\operatorname{Gal}(A, B)$ stand in a one-to-one correspondence with certain semi-ideals of $A \times B$. Let us say that $E \subseteq A \times B$ is a $G$-ideal of $A \times B$ when

(a) $E \in \mathcal{D}(A \times B)$ (the set of all down-sets of $A \times B$ ), that is,

$$
\downarrow E:=\{(x, y) \mid(x, y) \leq(a, b) \text { for some }(a, b) \in E\}
$$

coincides with $E$;

(b) $(\{x\} \times S \subseteq E \Rightarrow(x, \bigvee S) \in E)$ and $(S \times\{y\} \subseteq E \Rightarrow(\bigvee S, y) \in E)$. 
Note that the case $S=\emptyset$ in (b) implies that every G-ideal contains the set $\downarrow(1,0) \cup \downarrow(0,1)$. We denote by $A \otimes B$ the set of all G-ideals of $A \times B$, partially ordered by inclusion. This is clearly a complete lattice, because the intersection of G-ideals is always a G-ideal. In summary, $A \otimes B \cong \operatorname{Gal}(A, B)$ is the tensor product in the category of complete join-semilattices.

For every $a \in A$ and $b \in B$,

$$
\begin{aligned}
p_{a, b}: A & \longrightarrow \\
x & \longmapsto \begin{cases}1 & \text { if } x=0 \\
b & \text { if } 0<x \leq a \\
0 & \text { if } x \leq \leq a .\end{cases}
\end{aligned}
$$

is a Galois map. These maps generate $\operatorname{Gal}(A, B)$ by joins, that is, every $f \in$ $\operatorname{Gal}(A, B)$ is a join of some family $\left\{p_{a_{i}, b_{i}}\right\}_{i \in I} \subseteq \operatorname{Gal}(A, B)[22]$. Under the isomorphism $\operatorname{Gal}(A, B) \cong A \otimes B$, the Galois map $p_{a, b}$ corresponds to the least G-ideal containing $(a, b)$, that is,

$$
a \otimes b:=\downarrow(a, b) \cup \downarrow(1,0) \cup \downarrow(0,1) .
$$

This is called a pure tensor. The tensor product construction above gives us the construction of products in the category of locales. Indeed, $A \otimes B$ is a frame whenever $A$ and $B$ are frames and $\left(A \otimes B, u_{1}, u_{2}\right)$ is the coproduct of $A$ and $B$ in the category of frames, where

$$
\begin{aligned}
u_{1}: A & \longrightarrow A \otimes B \\
a & \longmapsto a \otimes 1
\end{aligned}
$$

and

$$
\begin{aligned}
u_{2}: B & \longrightarrow A \otimes B \\
b & \longmapsto 1 \otimes b .
\end{aligned}
$$

\section{The Galois composition}

From now on, for complete lattices $A$ and $B$, let $\operatorname{Ant}(A, B)$ denote the set of all antitone maps from $A$ to $B$. This is, clearly, a complete lattice (meets and joins are just defined pointwise).

For $f \in \operatorname{Ant}(A, B)$ and $g \in \operatorname{Ant}(B, C)$ let $E_{f, g}$ denote the G-ideal

$$
\bigvee\{a \otimes c \in A \otimes C \mid \exists b \in B \backslash\{0\}: f(a) \geq b \text { and } g(b) \geq c\} .
$$

The Galois composition $g \circ f: A \rightarrow C$ is defined by

$$
(g \circ f)(a):=\bigvee\left\{c \in C \mid(a, c) \in E_{f, g}\right\} .
$$

Proposition 3.1. For every $f \in \operatorname{Ant}(A, B)$ and $g \in \operatorname{Ant}(B, C), g \circ f \in \operatorname{Gal}(A, C)$. 
Proof. Let us show that $(g \circ f)\left(\bigvee_{i \in I} a_{i}\right)=\bigwedge_{i \in I}(g \circ f)\left(a_{i}\right)$. Since G-ideals are downwards closed, $g \circ f$ is clearly order-reversing. Therefore $(g \circ f)\left(\bigvee_{i \in I} a_{i}\right) \leq$ $(g \circ f)\left(a_{i}\right)$ for every $i \in I$. On the other hand, let $c \leq(g \circ f)\left(a_{i}\right)$ for every $i \in I$. By the definition of G-ideal, we have, for each $i \in I$,

$$
\left(a_{i}, \bigvee\left\{d \in C \mid\left(a_{i}, d\right) \in E_{f, g}\right\}\right) \in E_{f, g}
$$

that is, $\left(a_{i}, g \circ f\left(a_{i}\right)\right) \in E_{f, g}$. Thus $\left(a_{i}, c\right) \in E_{f, g}$ and, consequently, $\left(\bigvee_{i \in I} a_{i}, c\right) \in$ $E_{f, g}$, which implies $c \leq(g \circ f)\left(\bigvee_{i \in I} a_{i}\right)$. Hence $(g \circ f)\left(\bigvee_{i \in I} a_{i}\right)=\bigwedge_{i \in I}(g \circ f)\left(a_{i}\right)$, which means that $g \circ f \in \operatorname{Gal}(A, C)$.

For a categorical description of the Galois composition, that justifies the "correctness" of the chosen definition, see [7].

The following fact about $\operatorname{Gal}(A, B)$ will be decisive in the sequel:

Lemma 3.2. Let $f \in \operatorname{Gal}(A, B)$ and $E \in \mathcal{D}(A \times B)$ satisfy

$$
(a, b) \in E \Rightarrow b \leq f(a) .
$$

Then

$$
(a, b) \in\langle E\rangle \Rightarrow b \leq f(a)
$$

where $\langle E\rangle$ denotes the $G$-ideal generated by $E$.

Proof. Consider the non-empty set

$$
\mathcal{F}:=\{F \in \mathcal{D}(A \times B) \mid E \subseteq F \subseteq\langle E\rangle,(a, b) \in F \Rightarrow b \leq f(a)\} .
$$

If $F \in \mathcal{F}$ then also

$$
F_{0}:=\{(a, \bigvee S) \mid\{a\} \times S \subseteq F\} \cup\{(\bigvee S, b) \mid S \times\{b\} \subseteq F\}
$$

is in $\mathcal{F}$ :

Let $(a, b) \in F_{0}$. If $(a, b)=(a, \bigvee S)$ for some $S$ with $\{a\} \times S \subseteq F$, then $s \leq f(a)$ for every $s \in S$. Thus $\bigvee S \leq f(a)$. On the other hand, if $(a, b)=(\bigvee S, b)$ for some $S$ with $S \times\{b\} \subseteq F$, then $b \leq f(s)$ for every $s \in S$. This implies $b \leq \wedge f(S)=$ $f(\bigvee S)=f(a)$.

Besides, for any non-empty $\mathcal{H} \subseteq \mathcal{F}, \bigcup_{H \in \mathcal{H}} H \in \mathcal{F}$. Therefore $T:=\bigcup_{F \in \mathcal{F}} F$ belongs to $\mathcal{F}$, that is, $\mathcal{F}$ has a largest element $T$. But then, as we proved above, $T_{0}$ also belongs to $\mathcal{F}$ so $T=T_{0}$, that is, $T$ is a G-ideal. Hence $\langle E\rangle=T \in \mathcal{F}$ which means that $b \leq f(a)$ whenever $(a, b) \in\langle E\rangle$. 


\section{The quantale of antitone maps}

In order to prove that $\circ$ is a quantic multiplication on $\operatorname{Gal}(L, L)$ we introduce the following auxiliary operation on antitone maps:

Let $f \in \operatorname{Ant}(A, B)$ and $g \in \operatorname{Ant}(B, C)$; the antitone composition $g \cdot f: A \rightarrow C$ is defined by

$$
(g \cdot f)(a):=\bigvee\{c \in C \mid \exists b \in B \backslash\{0\}: f(a) \geq b \text { and } g(b) \geq c\} .
$$

Remarks 4.1. (1) It is obvious that $(g \cdot f)(a)=\bigvee\{g(b) \mid b \in B \backslash\{0\}, b \leq f(a)\}$ and $g \cdot f \in \operatorname{Ant}(A, C)$.

(2) It is also easy to see that $(g \cdot f)(a)$ coincides with

$$
\bigvee\{c \in C \mid(a, c) \in\{(x, z) \in A \times C \mid \exists b \in B \backslash\{0\}: f(x) \geq b \text { and } g(b) \geq z\}\} \text {. }
$$

Thus $g \cdot f \leq g \circ f$. As we shall see in Proposition 6.4, $g \circ f$ is the least Galois map containing $g \cdot f$.

Note that $g \cdot f_{1} \leq g \cdot f_{2}$ whenever $f_{1} \leq f_{2}$.

Lemma 4.2. Let $f \in \operatorname{Ant}(A, B)$ and $g \in \operatorname{Ant}(B, C)$. If $C$ is a frame then $c \leq$ $(g \cdot f)(a)$ if and only if $c=\bigvee S$ for some $S \subseteq C$ such that for each $s \in S$ there exists $b \in B \backslash\{0\}$ satisfying $f(a) \geq b$ and $g(b) \geq s$.

Proof. Let $C$ be a frame. If $c \leq(g \cdot f)(a)$ then, using Remark 4.1(1), we have

$$
\begin{aligned}
c & =c \wedge(g \cdot f)(a) \\
& =c \wedge \bigvee\{g(b) \mid b \in B \backslash\{0\}, b \leq f(a)\} \\
& =\bigvee\{c \wedge g(b) \mid b \in B \backslash\{0\}, b \leq f(a)\},
\end{aligned}
$$

where, for each such $b, c \wedge g(b) \leq g(b)$ and $b \leq f(a)$.

Conversely, let $c=\bigvee_{i \in I} s_{i}$ where, for each $i \in I$, there exists $b_{i} \in B \backslash\{0\}$ such that $s_{i} \leq g\left(b_{i}\right)$ and $b_{i} \leq f(a)$. Then, obviously, $s_{i} \leq(g \cdot f)(a)$ and, consequently, $c \leq(g \cdot f)(a)$.

In order to conclude the associativity of the antitone composition we have to restrict ourselves to frames.

Proposition 4.3. Let $f \in \operatorname{Ant}(A, B), g \in \operatorname{Ant}(B, C)$ and $h \in \operatorname{Ant}(C, D)$. If $C$ and $D$ are frames then $(h \cdot g) \cdot f=h \cdot(g \cdot f)$.

Proof. First, let us show that $((h \cdot g) \cdot f)(a) \leq(h \cdot(g \cdot f))(a)$, for every $a \in A$, that is, $\bigvee\{d \in D \mid \exists b \in B \backslash\{0\}: f(a) \geq b$ and $(h \cdot g)(b) \geq d\} \leq(h \cdot(g \cdot f))(a)$. Of course, it suffices to ensure that $d \leq(h \cdot(g \cdot f))(a)$ whenever $f(a) \geq b \neq 0$ and $(h \cdot g)(b) \geq d$. So let $f(a) \geq b \neq 0$ and $(h \cdot g)(b) \geq d$. Then, since $D$ is a frame, we may apply Lemma 4.2 and write $d$ as a join $\bigvee_{i \in I} d_{i}$ of a family $\left\{d_{i} \mid i \in I\right\} \subseteq D$ for which there 
exist $c_{i} \in C \backslash\{0\}, i \in I$, satisfying $g(b) \geq c_{i}$ and $h\left(c_{i}\right) \geq d_{i}$. Then $(g \cdot f)(a) \geq c_{i} \neq 0$ and $h\left(c_{i}\right) \geq d_{i}$ for every $i \in I$, and we may conclude that $(h \cdot(g \cdot f))(a) \geq d_{i}$ for every $i \in I$; consequently, $(h \cdot(g \cdot f))(a) \geq \bigvee_{i \in I} d_{i}=d$.

Finally, let us prove the reverse inequality $((h \cdot g) \cdot f)(a) \geq(h \cdot(g \cdot f))(a)$. We need to show that $d \leq((h \cdot g) \cdot f)(a)$ whenever $(g \cdot f)(a) \geq c \neq 0$ and $h(c) \geq d$. Again by Lemma 4.2, since $C$ is a frame, we may write $c=\bigvee_{i \in I} c_{i}$ for $c_{i} \in C \backslash\{0\}$ satisfying $f(a) \geq b_{i}$ and $g\left(b_{i}\right) \geq c_{i}$ for some $b_{i} \in B \backslash\{0\}$. But $d \leq h(c) \leq h\left(c_{i}\right)$ so $d \leq(h \cdot g)\left(b_{i}\right)$ for each $i \in I$. Hence $d \leq((h \cdot g) \cdot f)(a)$.

Theorem 4.4. If $L$ is a frame then $(\operatorname{Ant}(L, L), \cdot)$ is a quantale.

Proof. By Remark 4.1(3), we have immediately that $g \cdot\left(\bigvee_{i \in I} f_{i}\right) \geq \bigvee_{i \in I}\left(g \cdot f_{i}\right)$. Let us check that $\left(g \cdot\left(\bigvee_{i \in I} f_{i}\right)\right)(a) \leq\left(\bigvee_{i \in I}\left(g \cdot f_{i}\right)\right)(a)$, for every $a \in L$, that is,

$$
\bigvee\left\{c \in L \mid \exists b \in L \backslash\{0\}: \bigvee_{i \in I} f_{i}(a) \geq b \text { and } g(b) \geq c\right\}
$$

is equal or less than

$$
\bigvee_{i \in I} \bigvee\left\{c \in L \mid \exists b \in L \backslash\{0\}: f_{i}(a) \geq b \text { and } g(b) \geq c\right\}
$$

So, consider $c \in L$ for which there exists $b \in L \backslash\{0\}$ satisfying $b \leq \bigvee_{i \in I} f_{i}(a)$ and $c \leq g(b)$. Then $b=b \wedge \bigvee_{i \in I} f_{i}(a)=\bigvee_{i \in I}\left(b \wedge f_{i}(a)\right)$. This implies the existence of $i \in I$ for which $b \wedge f_{i}(a) \neq 0$. Denoting this element $b \wedge f_{i}(a)$ by $b^{\prime}$ we have $c \leq g(b) \leq g\left(b^{\prime}\right)$ and $0 \neq b^{\prime} \leq f_{i}(a)$. This proves $\left(g \cdot\left(\bigvee_{i \in I} f_{i}\right)\right)(a) \leq\left(\bigvee_{i \in I}\left(g \cdot f_{i}\right)\right)(a)$.

Similarly,

$$
\left(\bigvee_{i \in I} g_{i}\right) \cdot f=\bigvee_{i \in I}\left(g_{i} \cdot f\right) \text {. }
$$

This, together with Proposition 4.3, shows that $(\operatorname{Ant}(L, L), \cdot)$ is a quantale.

\section{Quantic nuclei and prenuclei}

We need some facts about quotients of quantales, most of which can be found in [16] or [21].

A quantic nucleus [16] on a quantale $Q$ is a closure operator $j: Q \rightarrow Q$ such that $j(x) \cdot j(y) \leq j(x \cdot y)$, for all $x, y \in Q$. Note that if $j$ is a quantic nucleus, then

$$
j(x \cdot y)=j(x \cdot j(y))=j(j(x) \cdot y)=j(j(x) \cdot j(y)),
$$

for all $x, y \in Q$. The set of $j$-closed elements

$$
Q_{j}:=F i x(j)=\{x \in Q \mid j(x)=x\}
$$

is closed under $\bigwedge$ and hence is complete (of course, this is true for any closure operator $j$ on a complete lattice $Q$ ). In this case, $j$ being a quantic nucleus on a 
quantale $Q, Q_{j}$ is a quantale with joins $\bigvee^{j} x_{i}=j\left(\bigvee x_{i}\right)$ and multiplication $x \cdot{ }_{j} y=$ $j(x \cdot y)$. Indeed,

$\left(x \cdot{ }_{j} y\right) \cdot{ }_{j} z=j(j(x \cdot y) \cdot z)=j((x \cdot y) \cdot z)=j(x \cdot(y \cdot z))=j(x \cdot j(y \cdot z))=x \cdot{ }_{j}\left(y \cdot{ }_{j} z\right)$,

$x \cdot{ }_{j}\left(\bigvee^{j} y_{i}\right)=j\left(x \cdot j\left(\bigvee y_{i}\right)\right)=j\left(x \cdot \bigvee y_{i}\right)=j\left(\bigvee\left(x \cdot y_{i}\right)\right) \leq j\left(\bigvee\left(j\left(x \cdot y_{i}\right)\right)\right)=\bigvee^{j}\left(x \cdot{ }_{j} y_{i}\right)$

and, consequently, $x \cdot \cdot_{j}\left(\bigvee^{j} y_{i}\right)=\bigvee^{j}\left(x \cdot{ }_{j} y_{i}\right)$; similarly, $\left(\bigvee^{j} x_{i}\right) \cdot{ }_{j} y=\bigvee^{j}\left(x_{i} \cdot{ }_{j} y\right)$.

Furthermore, the map $j: Q \rightarrow Q_{j}$ is a homomorphism of quantales and every surjective quantale homomorphism arises in this manner, up to isomorphism [16].

In the next section, in order to conclude that $(\operatorname{Gal}(L, L), \circ)$ is a quantale, we will define a quantic nucleus generated by certain data. As a useful intermediate step we introduce the following notion, which generalizes Banaschewski's definition of a (localic) prenucleus [1, 2]:

Definition 5.1. A quantic prenucleus on a quantale $L$ is a map $j_{0}: Q \rightarrow Q$ such that, for all $x, y \in Q$,

(1) $x \leq j_{0}(x)$,

(2) if $x \leq y$ then $j_{0}(x) \leq j_{0}(y)$,

(3) $j_{0}(x) \cdot y \leq j_{0}(x \cdot y)$ and $x \cdot j_{0}(y) \leq j_{0}(x \cdot y)$.

It follows immediately from the first two conditions that $Q_{j_{0}}=F i x\left(j_{0}\right)$ is a closure system and the associated closure operator is given by

$$
j(x):=\bigwedge\left\{y \in Q_{j_{0}} \mid x \leq y\right\} .
$$

Lemma 5.2. The closure operator $j$ is a quantic nucleus.

Proof. Let us show that $j(x) \cdot y \leq j(x \cdot y)$ for all $x, y \in Q$. For this, consider the set

$$
\mathcal{E}:=\{z \in Q \mid x \leq z \leq j(x), z \cdot y \leq j(x \cdot y)\} .
$$

This is a non-empty set (since $x \in \mathcal{E}$ ) and $j_{0}(z) \in \mathcal{E}$ whenever $z \in \mathcal{E}$ by the properties of $j_{0}$ and $j$. Moreover, for any non-void $\mathcal{F} \subseteq \mathcal{E}, \bigvee_{z \in \mathcal{F}} z$ belongs to $\mathcal{E}$, by the distribution law of quantales. Therefore $\mathcal{E}$ has a largest element $\bar{z}:=\bigvee_{z \in \mathcal{E}} z$. But then $j_{0}(\bar{z}) \in \mathcal{E}$ thus $\bar{z}=j_{0}(\bar{z})$ i.e., $\bar{z} \in Q_{j_{0}}$. Then $j(x)=\bar{z} \in \mathcal{E}$ and, consequently, $j(x) \cdot y \leq j(x \cdot y)$.

By symmetry, $x \cdot j(y) \leq j(x \cdot y)$. Hence $j(x) \cdot j(y) \leq j(x \cdot j(y)) \leq j^{2}(x \cdot y)=j(x \cdot y)$ as claimed.

Remarks 5.3. (1) It can be also proved that the quantale homomorphism $j: Q \rightarrow$ $Q_{j_{0}}$ is universal among all quantale homomorphisms $h: Q \rightarrow Q^{\prime}$ for which $h(x)=$ $h\left(j_{0}(x)\right)$ for all $x \in Q$.

(2) The quantic nucleus $j$ may alternatively be described as the stable transfinite iterate of $j_{0}$, and then the Lemma can be proved by transfinite induction. 


\section{The quantale of Galois connections}

For each $f \in \operatorname{Ant}(A, B)$ let us define $j_{0}(f): A \rightarrow B$ by

$$
j_{0}(f)(a):=\bigvee\{b \in B \mid \exists S \subseteq A: \bigvee S=a \text { and } f(s) \geq b \text { for every } s \in S\}
$$

Clearly, $j_{0}$ is an extensive operator, that is, $j_{0}(f) \geq f$. The motivation for the definition of $j_{0}$ comes from the following proposition asserting that Galois maps are precisely the fixed points of $j_{0}$.

Proposition 6.1. For each $f \in \operatorname{Ant}(A, B)$ we have:

(1) If $A$ is a frame then $j_{0}(f) \in \operatorname{Ant}(A, B)$.

(2) $j_{0}(f)=f$ if and only if $f \in \operatorname{Gal}(A, B)$.

Proof. (1) Let $a \leq a^{\prime}$ in $A$. We need to show that $j_{0}(f)\left(a^{\prime}\right) \leq j_{0}(f)(a)$, that is,

$$
\begin{aligned}
\bigvee\{b & \left.\in B \mid \exists S \subseteq A: \bigvee S=a^{\prime} \text { and } f(s) \geq b \text { for every } s \in S\right\} \\
& \leq \bigvee\{b \in B \mid \exists S \subseteq A: \bigvee S=a \text { and } f(s) \geq b \text { for every } s \in S\}
\end{aligned}
$$

So, consider $b$ in the first join, that is, $b \in B$ satisfying $b \leq f(s)$ for every $s$ in some $S \subseteq A$ for which $\bigvee S=a^{\prime}$. Then $a=a \wedge a^{\prime}=a \wedge \bigvee S=\bigvee_{s \in S}(a \wedge s)$, by the frame distributive law. Thus, since $b \leq f(s) \leq f(a \wedge s), b$ is also in the second join. (2) By the definition of $j_{0}$ it is clear that $j_{0}(f)(\bigvee S) \geq \bigwedge f(S)$ for every $S \subseteq A$. Therefore, if $j_{0}(f)=f$ then $f(\bigvee S) \geq \bigwedge f(S)$. Thus, since $f(\bigvee S) \leq \wedge f(S)$ is always true, $f(\bigvee S)=\bigwedge f(S)$, that is, $f$ is a Galois map.

Conversely, let $f \in \operatorname{Gal}(A, B)$ and $a \in A$. For each $b$ in

$$
\{b \in B \mid \exists S \subseteq A: \bigvee S=a \text { and } f(s) \geq b \text { for every } s \in S\}
$$

we have $b \leq \bigwedge f(S)=f(\bigvee S)=f(a)$, which shows that $j_{0}(f)(a) \leq f(a)$. Hence $j_{0}(f) \leq f$. Then, immediately, $j_{0}(f)=f$ since $j_{0}$ is extensive.

Proposition 6.2. If $L$ is a frame then $j_{0}: \operatorname{Ant}(L, L) \rightarrow \operatorname{Ant}(L, L)$ is a quantic prenucleus.

Proof. Obviously $j_{0}$ is extensive and monotone. Let us prove that $\left(g \cdot j_{0}(f)\right)(x) \leq$ $\left(j_{0}(g \cdot f)\right)(x)$ for all $x \in L$ and $f, g \in \operatorname{Ant}(L, L)$.

So, consider $y \in L$ satisfying $j_{0}(f)(x) \geq z$ and $g(z) \geq y$ for some nonzero $z \in L$. Then

$$
\begin{aligned}
z & =z \wedge j_{0}(f)(x) \\
& =z \wedge \bigvee\{a \in L \mid \exists S \subseteq L: \bigvee S=x \text { and } f(s) \geq a \text { for every } s \in S\} \\
& =\bigvee\{z \wedge a \mid a \in L, \exists S \subseteq L: \bigvee S=x \text { and } f(s) \geq a \text { for every } s \in S\}
\end{aligned}
$$


The fact $z \neq 0$ implies the existence of $a \in L$ and $S \subseteq L$ such that $x=\bigvee S$, $f(s) \geq a$ for all $s \in S$ and $z \wedge a \neq 0$. Then $g(z \wedge a) \geq g(z) \geq y$ and, for each $s \in S, f(s) \geq a \geq z \wedge a \neq 0$. This means $(g \cdot f)(s) \geq y$ for every $s \in S$. Therefore $\left(j_{0}(g \cdot f)\right)(x) \geq y$.

The condition $j_{0}(g) \cdot f \leq j_{0}(g \cdot f)$ may be proved in a similar manner.

Then $\operatorname{Ant}(L, L)_{j_{0}}$ (which, by Proposition 6.1(2), coincides with $\left.\operatorname{Gal}(L, L)\right)$ is a closure system and, by Lemma 5.2, the associated closure operator $j: \operatorname{Ant}(L, L) \rightarrow$ $\operatorname{Ant}(L, L)$ given by

$$
j(f):=\bigwedge\{g \in \operatorname{Gal}(L, L) \mid f \leq g\}
$$

is a quantic nucleus. Hence, by Theorem 4.4 and the results on quantic nuclei of [16] recalled at the beginning of Section 5, we have:

Corollary 6.3. If $L$ is a frame then $\left(\operatorname{Gal}(L, L), \cdot{ }_{j}\right)$ is a quantale.

Now the conclusion that the operation $\cdot_{j}$ coincides with the Galois composition o introduced in Section 3 follows from the following general result:

Proposition 6.4. Let $A, B$ and $C$ be complete lattices. For every $f \in \operatorname{Ant}(A, B)$ and $g \in \operatorname{Ant}(B, C), j(g \cdot f)=g \circ f$.

Proof. By Remark 4.1(2), $g \cdot f \leq g \circ f$. Now let $h \in \operatorname{Gal}(A, C)$ satisfying $h \geq g \cdot f$. We need to show that $h \geq g \circ f$ in order to conclude the proof. Let $a \in A$. Since $(g \circ f)(a)=\bigvee\left\{c \in C \mid(a, c) \in E_{f, g}\right\}$, by Lemma 3.2 it suffices to check that $c \leq h(a)$ whenever $(a, c) \in\{(x, z) \in A \times C \mid \exists y \in B \backslash\{0\}: f(x) \geq y$ and $g(y) \geq z\}$, which is easy: if $(a, c)=(x, z)$ for such a pair $(x, z)$ then $c=z \leq g(y)$ and $0 \neq y \leq f(x)=f(a)$, which implies $c \leq(g \cdot f)(a) \leq h(a)$.

Corollary 6.5. If $L$ is a frame then $(\operatorname{Gal}(L, L), \circ)$ is a quantale.

\section{Applications}

For any set $X$, the power set $\mathcal{P}(X \times X)$ equipped with the usual composite relation $S \circ R$ is a quantale. This quantale of binary relations on $X$ is the basic structure on which the theory of uniform spaces of Weil [23] relies (the calculus of relations has also played an important rôle in the interaction between algebra and logic since the middle of the nineteenth century; a first adequate development of such algebras was given by de Morgan and Peirce).

Binary relations can be described by polarities (i.e., Galois connections between power sets) [5]. In fact, there is a one-to-one correspondence between relations $R \subseteq X \times X$ and Galois connections on $\mathcal{P}(X)$, that assigns to each relation $R$ the 
Galois connection $\left(R_{\forall}, R^{\forall}\right): \mathcal{P}(X) \rightarrow \mathcal{P}(X)$ defined by

$$
R_{\forall}(U):=\bigcap_{a \in U}\{b \in X \mid(a, b) \in R\}=\{b \in X \mid \forall a \in U(a, b) \in R\} \quad \text { for } U \subseteq X
$$

and

$$
R^{\forall}(V):=\bigcap_{b \in V}\{a \in X \mid(a, b) \in R\}=\{a \in X \mid \forall b \in V(a, b) \in R\} \quad \text { for } V \subseteq X .
$$

In particular, Weil entourages $E$ of $X$ (that is, reflexive binary relations on $X$ ) correspond to Galois connections $\left(E_{\forall}, E^{\forall}\right)$ satisfying $\{x\} \subseteq E_{\forall}(\{x\})$ for all $x \in X$ or, equivalently, $\bigcup\left\{A \subseteq X \mid A \subseteq E_{\forall}(A)\right\}=X$.

Similarly, binary relations may also be described by axialities, that is, residuated pairs between power sets (see [5] for the details).

Therefore the classical description of uniform spaces, introduced by Weil in terms of entourages, may be immediately formulated in terms of polarities and axialities. In all these entourage-like approaches to uniform structures the main tool is a certain quantic structure. The theory of uniform spaces of Weil can be generalized to the pointfree context of frames and locales (see [20] for a survey on this generalization). Of course, it seems desirable to have characterizations of uniform frames that are analogous to those of uniformities which are given by polarities and axialities. In order to pursue this goal one needs "good" quantic structures for $\operatorname{Gal}(L, L)$ and $\operatorname{Gal}\left(L, L^{o p}\right)$. The former is presented in this paper; we get it precisely in the context that we need it: when $L$ is a frame. In [7] it is shown how to apply it to get a new (equivalent) approach to uniform frames. The latter is the well-known quantale of residuated maps ([5], [21]). By applying it to the study of uniform frames, as it is shown in [7], one gets precisely Fletcher-Hunsaker-Lindgren's approach [8]. This puts some light on the nature of this approach: Galois connections rather than entourages are at its root.

We can say more; the equivalence between the aproaches to frame uniformities presented in [8] and [19] is a special case of the following very general phenomenon on Galois connections, relating the elements of the quantales $\operatorname{Gal}(L, L)$ and $\operatorname{Gal}\left(L, L^{o p}\right)$. For each $f \in \operatorname{Gal}(L, L)$ let $f^{\uparrow}: L \rightarrow L$ be defined by

$$
f^{\uparrow}(x):=\bigvee\{y \in L \mid y \wedge x \neq 0, y \leq f(y)\} .
$$

This is a residuated map on $L$ and $f \leq g$ implies $f^{\uparrow} \leq g^{\uparrow}$. Conversely, given $f \in \operatorname{Gal}\left(L, L^{o p}\right)$, define $f^{\downarrow}: L \rightarrow L$ by putting

$$
f^{\downarrow}(x):=\bigvee\{y \in L \mid(x, y) \in \bigvee\{a \otimes a \mid a \text { is } f \text {-small }\}\},
$$

where $a \in L$ is said to be $f$-small whenever $a \wedge b \neq 0$ implies $a \leq f(b)$. This way we get a Galois map $f^{\downarrow}$ on $L$ and $f \leq g$ implies $f^{\downarrow} \leq g^{\downarrow}$. Since $f^{\uparrow \downarrow}$ and $f^{\downarrow \uparrow}$ may differ from $f$, these correspondences do not establish a one-to-one correspondence 
between $\operatorname{Gal}(L, L)$ and $\operatorname{Gal}\left(L, L^{o p}\right)$. Nevertheless we have the following (cf. [7] for the details):

Let us say that a Galois map $f$ is symmetric [7] whenever $f^{+}=f$. It is straightforward to check that $f^{\downarrow}$, for every residuated $f$, is symmmetric. Similarly, a symmetric residuated map [7] is a residuated map $f$ for which

$$
f(x) \wedge y \neq 0 \Leftrightarrow x \wedge f(y) \neq 0 \text { for all } x, y \in L .
$$

The map $f^{\uparrow}$, for every Galois $f$, is symmetric. Let us also extend the concepts of polarity and axiality to any frame $L$ by saying that $f \in \operatorname{Gal}(L, L)$ is a polarity of $L$ whenever $\bigvee\{x \in L \mid x \leq f(x)\}=1$, and that $f \in \operatorname{Gal}\left(L, L^{o p}\right)$ is an axiality of $L$ if $\bigvee\{x \in L \mid x$ is $f$-small $\}=1$. Then, for every symmetric polarity $f$ of $L$,

$$
f \leq(f \circ f)^{\uparrow \downarrow} \text { and } f^{\uparrow \downarrow} \leq f \circ f
$$

and, for every symmetric axiality $f$ of $L$,

$$
f \leq(f \circ f \circ f)^{\downarrow \uparrow} \text { and } f^{\downarrow \uparrow} \leq f[7] .
$$

This means that, in the presence of the square-refinement axiom (which asserts that for every $f$ in the structure there exists $g$ in the structure satisfying $g \circ g \leq f$ ) and the axiom of symmetry, we get a bijection. More precisely, the correspondences $f \mapsto f^{\uparrow}$ and $f \mapsto f^{\downarrow}$ establish an isomorphism between the filters of polarities of $L$ satisfying the symmetry and square-refinement axioms and the filters of axialities of $L$ satisfying the symmetry and square-refinement axioms. This fact about Galois connections explains why the somewhat different approaches to uniform frames presented in [8] and [19] are equivalent. The details can be found in [7].

Acknowledgements. I would like to thank Pedro Resende for his valuable suggestions regarding the presentation of the results of this paper. Thanks go also to the referee for several helpful comments and suggestions that improved the readability of this paper and for alerting us for a mistake in a previous version of Lemma 3.2.

\section{REFERENCES}

[1] B. Banaschewski, Another look at the localic Tychonoff Theorem, Comment. Math. Univ. Carolin. 29 (1988), 647-656.

[2] B. Banaschewski, On certain localic nuclei, Cahiers Topologie Géom. Différentielle Catég. 35 (1994), 227-237.

[3] G. Birkhoff, Lattice Theory, Amer. Math. Soc. Colloquium Publications, Providence, Rhode Island, 1st edition, 1940 (3rd edition 1967).

[4] T. S. Blyth and M. F. Janowitz, Residuation Theory, International Series of Monographs in Pure and Applied Mathematics, Vol. 102, Pergamon Press, 1972.

[5] M. Erné, J. Koslowski, A. Melton and G. E. Strecker, A primer on Galois connections, in: Papers on general topology and applications (Madison, WI, 1991), Ann. New York Acad. Sci., Vol. 704, 1993, pp. 103-125. 
[6] C. J. Everett, Closure operators and Galois theory in lattices, Trans. Amer. Math. Soc. 55 (1944), 514-525.

[7] M. J. Ferreira, J. Picado, The Galois approach to uniform structures, preprint, University of Coimbra, submitted.

[8] P. Fletcher, W. Hunsaker,and W. Lindgren, A functional approach to uniform and quasi-uniform frames, in: Topology with Applications, Bolyai Soc. Math. Studies, Vol. 4, Szekszárd, 1993, p. 217-222.

[9] R. Giles and H. Kummer, A non-commutative generalization of topology, Indiana Univ. Math. J. 21 (1971), 91-102.

[10] G. Grätzer and F. Wehrung, Tensor products of semilattices with zero, revisited, J. Pure Appl. Algebra 147 (2000), 273-301.

[11] H. Herrlich and M. Hušek, Galois connections categorically, J. Pure Appl. Algebra 68 (1990), 165-180.

[12] J. Isbell, Atomless parts of spaces, Math. Scand. 31 (1972), 5-32.

[13] P. T. Johnstone, Stone Spaces, Cambridge University Press, Cambridge, 1982.

[14] A. Joyal and M. Tierney, An extension of the Galois Theory of Grothendieck, Mem. Amer. Math. Soc. 309, 1984.

[15] C. J. Mulvey, \&, Rend. Circ. Mat. Palermo (2) Suppl. No. 12 (1986), 99-104.

[16] S. Niefield and K. Rosenthal, Constructing locales from quantales, Math. Proc. Camb. Phil. Soc. 104 (1988), 215-234.

[17] Ore, O., Galois connexions, Trans. Amer. Math. Soc. 55 (1944), 493-513.

[18] J. Paseka and J. Rosický, Quantales, in: Current Research in Operational Quantum Logic, B.Coecke, D.J. Moore and A. Wilce (Eds.), Fundamental Theories of Physics-series, Kluwer Academic Publishers, 2000, pp. 245-262.

[19] J. Picado, Weil uniformities for frames, Comment. Math. Univ. Carolinae 36 (1995), 357-370.

[20] J. Picado, Structured frames by Weil entourages, Appl. Categ. Struct. 8 (2000), 351-366.

[21] K. Rosenthal, Quantales and their applications, Pitman Research Notes in Mathematics 234, Longman, 1990.

[22] Z. Shmuely, The structure of Galois connections, Pacific J. Math. 54 (1974), 209-225.

[23] A. Weil, Sur les Espaces à Structure Uniforme et sur la Topologie Générale, Publications de l'Institute Mathématique de l'Université de Strasbourg, Hermann, Paris, 1938.

Jorge PicAdo

Departamento de Matemática, Universidade de Coimbra, 3001-454 Coimbra, Portugal

e-mail: picado@mat.uc.pt

To access this journal online:
http://www.birkhauser.ch

